\title{
sit
}

\section{Selección natural y condicionamiento de operantes: una crítica a la analogía} de Fodor y Piattelli-Palmarini

Julio Torres Meléndez

\begin{abstract}
电
RESUMEN

Me propongo examinar críticamente la analogía entre selección natural y condicionamiento operante que Fodor y Piattelli-Palmarini utilizan para sostener que los mismos argumentos que desacreditan la teoría conductista desacreditan la teoría de la selección natural. Estas teorías no son independientes ni conceptual ni históricamente y, por eso, no es razonable hacer una analogía entre ambas con las intenciones que se proponen Fodor y Piattelli-Palmarini. La selección natural es una condición causal del mecanismo de condicionamiento operante, motivo por el cual la refutación de la teoría del condicionamiento no puede ser una razón para la refutación de la teoría de la selección natural.
\end{abstract}

Palabras-Glave • Selección natural. Condicionamiento operante. Skinner. Fodor. Piattelli-Palmarini.

\section{INTRODUGGIÓN}

Me propongo en este artículo discutir la analogía entre selección natural y condicionamiento de conductas operantes que Jerry Fodor y Massimo Piattelli-Palmarini desarrollan para argumentar que la teoría darwiniana es una teoría fracasada (cf. FP, 2010). Fodor y Piattelli-Palmarini pretenden hacer un ataque destructivo a la teoría de la evolución dirigido sólo a la selección natural y no al supuesto de que la diversidad de la vida tiene un origen común. No ponen en duda que las relaciones de semejanza estructural que cruzan las diversas especies, la unidad de tipo de los formalistas predarwinianos, se expliquen por el supuesto evolucionista del ancestro común, pero niegan que la selección natural sea el proceso que explique la adaptación y la diversidad orgánica. Los autores pretenden que la selección natural carece del poder explicativo que Darwin y los evolucionistas actuales le adscriben. La explicación evolucionista es comparable, según Fodor y Piattelli-Palmarini, a la explicación histórica, en donde, de acuerdo con la interpretación anti-positivista, no hay leyes, no hay generalizaciones acerca de hechos históricos que proporcionen una explicación universal con 
fuerza contra-fáctica. La explicación darwiniana, como la explicación histórica, no sería más que una narración imaginativa meramente plausible para un conjunto de hechos singulares que no pueden tener una explicación legiforme, dado que no habría ningún trasfondo causal común a los procesos que la teoría de la selección natural pretende explicar.

La recepción del libro de Fodor y Piattelli-Palmarini ha sido en general crítica, si no francamente hostil. Los filósofos de la biología han defendido el carácter explicativo de la selección natural pero no se han detenido en discutir la analogía entre selección natural y condicionamiento de conductas operantes con que se introducen los argumentos de los autores en contra de la teoría darwiniana. Elliott Sober, en su artículo de 2010, no hace mención a ella, ni tampoco lo hace Antonio Diéguez (2012), cuando examina críticamente las afirmaciones de Fodor y Piattelli-Palmarini acerca de la selección natural. José Díez y Pablo Lorenzano tampoco abordan críticamente la analogía en su extenso ensayo en defensa del poder explicativo de la teoría darwiniana y proponen, en cambio, una analogía diferente, la analogía entre selección natural y mecánica clásica, para mostrar que la singularidad de la explicación por selección natural no es muy distinta de la que encontramos en la segunda ley de la mecánica de newton (cf. Díez \& Lorenzano, 2013). Peter Godfrey-Smith (2010), en su reseña del libro de Fodor y Piattelli-Palmarini, aborda la analogía pero su interés radica, básicamente, en mostrar que la intención de los autores es poner en el mismo nivel de relevancia histórica, por un lado, la célebre refutación que hiciera Noam Chomsky (1980 [1959]) de la teoría conductista y, por otro, el propio intento de refutación que ellos hacen de la teoría evolucionista. Respecto de la función argumentativa del símil, Godfrey-Smith (2010) se contenta con sostener que la analogía no es tan profunda y que el hecho de que la teoría conductista haya sido ampliamente abandonada no implica que la teoría de la evolución también lo sea.

Por mi parte, sostengo que es valioso explorar la analogía entre explicación conductista y explicación por selección, dado que Fodor y Piattelli-Palmarini piensan que ella ilumina aspectos fundamentales de la precariedad de la explicación darwiniana. En la investigación filosófica, las analogías tienen usualmente una función positiva, nos permiten ver con claridad aspectos del mundo del que desconocemos sus mecanismos causales, ya sea por la complejidad de su naturaleza o por la indigencia de nuestra formación teórica. ${ }^{\mathbf{1}}$ El uso que Fodor y Piattelli-Palmarini hacen es distinto, proponen una analogía con la intención de socavar los valores epistémicos de la teoría de la selección natural. De acuerdo con ellos, si ambas teorías son análogas, entonces los

1 Considérese, por ejemplo, el extendido uso que hace Ludwig Wittgenstein de analogías y figuras como medio de aclaración conceptual (cf. Wittgenstein, 2003). 
mismos argumentos que han sido decisivos, a partir de fines de 1950, en contra del conductismo, deberían ser también decisivos en contra de la teoría de la selección natural. La explicación de la persistencia histórica de la teoría de la selección natural no tiene que ver con sus valores científicos o epistémicos, sino que la explicación es más bien externa o cultural: "los biólogos evolucionistas simplemente no han leído mucho acerca de la historia de las teorías conductistas del aprendizaje" (FP, 2010, p. xvi).

Me propongo mostrar que hay razones conceptuales por las cuales la analogía fracasa en su doble pretensión. Argumentaré que la teoría conductista del aprendizaje está estrechamente vinculada a la teoría de la selección natural. La propuesta de que hay una fuerte vinculación entre ambas teorías parece inicialmente fortalecer la intención de la analogía de Fodor y Piattelli-Palmarini, pero no es así, pues no se trata aquí de que la lógica explicativa de ambas teorías sea semejante, sino de una relación de dependencia causal entre los procesos de selección natural y condicionamiento operante. Mi argumentación distingue dos aspectos en esa relación de dependencia:

(1) La relación propiamente histórica que muestra cómo se establece esta dependencia causal: la teoría de la selección natural no es solo un elemento en el contexto cultural en donde surge la teoría del condicionamiento operante, la teoría de la selección natural constituye una condición histórica que hizo posible la teoría del condicionamiento operante.

(2) La naturaleza de la conexión lógica de estas teorías dada su relación de dependencia causal. Me referiré en lo que sigue a este segundo aspecto, abordaré el primero en la sección 3.

Independientemente de sus logros explicativos, la teoría conductista del aprendizaje supone la fuerza explicativa de la teoría de la selección natural, es decir, la selección natural constituye una condición causal para los procesos de aprendizaje por condicionamiento operante y por esta razón constituye, desde el punto de vista lógico, una condición necesaria para la teoría del condicionamiento operante. La relación lógica que hay entre selección natural y condicionamiento operante es semejante a la relación que hay entre selección natural y darwinismo social, una teoría que supone a la selección natural como una condición causal para sustentar sus argumentaciones acerca de las bases biológicas de las diferencias entre los seres humanos, tal como lo ha expresado Gould (2009, p. 478). Pero hay una relación muy distinta entre, por ejemplo, selección natural y la ley de Fisher, según la cual la relación numérica entre sexos tiende a evolucionar hacia una proporción de 1:1. La ley de Fisher es una implicación contrastadora de la teoría de la selección natural, pues se deduce a partir de ella y otros supuestos o, desde el punto de vista kuhniano que defienden Díez y Lorenzano, que es 
una especialización (specialization) del principio de selección natural que incluye variables no contenidas o no especificadas en el principio (cf. Díez \& Lorenzano, 2013, p. 1168). De ahí que la demostración empírica de la falsedad de la ley de Fisher sería una razón para la refutación de la teoría de la selección natural o, si seguimos a Díez y Lorenzano, sería una anomalía que podría conducir eventualmente a su refutación. ${ }^{2}$ En cambio, el darwinismo social no se deduce de la teoría de la selección natural ni es, alternativamente, considerado un explanandum legitimado por la comunidad científica para constituirse en una especialización del principio de selección natural. El darwinismo social supone la selección natural como una condición causal. De la falsedad del darwinismo social no se obtienen entonces razones para la falsedad de la teoría de la selección natural, aunque de la falsedad de la selección natural sí se seguiría la falsedad del darwinismo social. El intento de refutar la teoría de la selección natural refutando el darwinismo social tendría entonces la forma de una falacia de la afirmación del consecuente:

$$
[(\sim \mathrm{SN} \rightarrow \sim \mathrm{DS}) \& \sim \mathrm{DS}] \rightarrow \sim \mathrm{SN}
$$

De la misma manera, si la selección natural es una condición causal del condicionamiento operante, el intento de desacreditar la teoría de la selección con los mismos argumentos que desacreditaron la teoría conductista es un proyecto que carece de fundamento argumentativo. Pretender refutar la teoría de la selección natural, asumiendo la refutación de la teoría del aprendizaje por condicionamiento operante, constituiría también una falacia de la afirmación del consecuente. Por cierto, Fodor y Piattelli-Palmarini no hacen esta argumentación, pues no asumen que la selección natural sea una condición necesaria del mecanismo de condicionamiento operante. Pero, como mostraré, se equivocan al ignorar completamente la conexión de prioridad causal y al tratar ambas teorías como independientes tanto histórica como conceptualmente.

Fodor y Piattelli-Palmarini sostienen que, a pesar de su descrédito, la teoría conductista del aprendizaje tiene una ventaja epistémica respecto de la teoría de la selección natural: supone un trasfondo de leyes psicológicas de asociación heredadas del empirismo británico que, aunque se han demostrado falsas, en principio, le pudieron

\footnotetext{
2 Entendida de este modo la relación entre la teoría de la selección natural y la ley de Fisher, queda abierta la posibilidad de que esta teoría tenga también especializaciones muy lejanas de sus explananda originales (primariamente, la frecuencia de un rasgo en una población) y surjan otros candidatos tales como la evolución de entidades sociales. Pero no creo que esto invalide mi argumentación, pues el mismo marco kuhniano que abre esta posibilidad exige que sea la comunidad científica involucrada la que valide esta extensión y no parece que sea este el caso, como lo admiten implícitamente Díez y Lorenzano (2013, p. 1145). Esto no supone adscribir a Díez y Lorenzano una posición acerca de si pueden o no haber especializaciones sociales de la teoría de la selección natural, solo es una afirmación acerca de la situación actual de la validación de la comunidad científica al respecto.
} 
haber dado sustento explicativo a la teoría ofreciendo un mecanismo causal que diera cuenta de cómo opera el filtro de conductas operantes. La teoría de la selección natural, en cambio, nunca ha tenido un candidato para constituirse en el trasfondo explicativo que diera cuenta de cómo opera la selección de rasgos de acuerdo a su valor para la sobrevivencia y la reproducción (cf. FP, 2010, p. 16). ${ }^{3}$ No abordaré esta última cuestión que ha sido la preocupación central de Sober (2010) y de Díez y Lorenzano (2013), mi objetivo a este respecto será solo argumentar que es errónea la afirmación de que la teoría del aprendizaje por condicionamiento operante suponga un trasfondo de leyes de asociación. El trasfondo causal de esta teoría del aprendizaje es justamente el proceso de selección natural.

\section{La analogía de Fodor y Piattelli-Palmarini}

De acuerdo con Fodor y Piattelli-Palmarini, la teoría de la selección natural y la teoría conductista del aprendizaje por condicionamiento operante son "virtualmente idénticas". Sus mecanismos, nos dicen, son esencialmente los mismos y están sometidos a idénticas constricciones acerca de cómo explicar los hechos del aprendizaje y de la evolución biológica. Prontamente, sin embargo, esta afirmación es matizada. Podría decirse que conviven en este libro dos versiones de la comparación entre selección natural y condicionamiento operante: una versión fuerte y una versión débil. Según la versión fuerte, ambas teorías son idénticas. Los autores llegan a sostener que "la explicación de Skinner del aprendizaje y la explicación de Darwin de la evolución son idénticas en todo excepto en el nombre" (FP, 2010, p. xvI).4 En la versión débil de la comparación se muestran, en cambio, aspectos en donde no hay coincidencia, por ejemplo, Fodor y Piattelli-Palmarini aluden al carácter local de las explicaciones evolucionistas, es decir, al hecho de que ellas quedan confinadas a los procesos de selección actuales y no a las contingencias futuras; en cambio, los procesos psicológicos

3 Se ha sostenido también la tesis exactamente contraria: "la teoría del condicionamiento operante no ofrece ningún mecanismo para explicar los cambios en la frecuencia de respuestas (...) en agudo contraste respecto de la teoría de la selección natural, pretende ser meramente descriptiva más bien que explicativa" (Catania \& Harnad, 1988, p. 417).

4 La analogía fuerte la hace antes Noam Chomsky en un diálogo académico en la Universidad del País Vasco en el año 2006 (publicado en Piattelli et al., 2009). Chomsky le atribuye al mismo Skinner, en Verbal behavior, la afirmación según la cual "la estructura lógica del condicionamiento, la teoría del reforzamiento, es la misma que la teoría darwiniana de la selección natural" (Piattelli et al., 2009, p. 39). Pero adviértase que Skinner solo dice "[q]ue hay un paralelo entre la selección natural y el condicionamiento operante. La selección de una respuesta instintiva por su efecto en promover la sobrevivencia de una especie es semejante (resembles) a la selección de una respuesta por medio del reforzamiento, excepto por la enorme diferencia en las escalas de tiempo. La similaridad se nota en la aparente intencionalidad (purposiveness) de ambas formas" (Skinner, 1957, p. 462). 
que intenta explicar la teoría del condicionamiento carecen de esta localidad, los animales con mente pueden no solo recordar sino que también anticipar, y este hecho, evidentemente, interviene en los fenómenos a explicar (FP, 2010, p. 11). Me propongo sostener que tanto la analogía fuerte como la débil son inapropiadas para representar correctamente la naturaleza de la teoría darwiniana y para determinar qué hechos dan cuenta de la persistencia histórica de esta teoría.

No pretendo afirmar que todos los argumentos de Fodor y Piattelli-Palmarini en contra de la teoría de la selección natural dependan de su analogía de la teoría conductista del aprendizaje, pero sí creo que debemos considerar con seriedad esta analogía, pues ella muestra la comprensión que los autores tienen de la teoría darwiniana y de esta comprensión depende la profundidad de sus críticas. La analogía porta también una fuerza propagandística que no debemos subestimar. Si la asociación cultural entre selección natural y conductismo resulta exitosa, buena parte del camino hacia la desacreditación de la teoría darwiniana ya está construido. Difícilmente encontraremos defensores hoy en día de la teoría del aprendizaje por condicionamiento operante como el substrato de las acciones humanas más complejas. Esto se explica, como es sabido, por la supresión que hace la teoría conductista de la función de los estados intencionales en la explicación de la acción y, también, porque los conductistas asumen que los resultados experimentales de procesos elementales de aprendizaje en animales no-humanos pueden ser extrapolados a la compleja estructura de la acción humana (cf. Chomsky, 1959; Taylor, 1964).

Me detendré a examinar críticamente la analogía débil. Sin embargo, es relevante mostrar que la analogía fuerte difícilmente es sostenible si se advierte que el mismo Skinner distingue el fenómeno del condicionamiento operante de la selección natural y de lo que llama "selección de ambientes sociales", estableciendo explícitamente las semejanzas y las diferencias entre estos fenómenos: "Hay notables similitudes [dice Skinner] entre selección natural, condicionamiento operante y evolución de ambientes sociales. No sólo todos ellos prescinden de un diseño creativo previo, de un propósito previo, ellos invocan la noción de sobrevivencia como un valor" (Skinner, 1976, p. 225). Más adelante, Skinner llegará a sostener que se trata aquí de tres niveles distintos de "variación y selección", los que son estudiados, respectivamente, por tres disciplinas distintas: la biología, la psicología y la antropología (Skinner, 1988b, p. 14). Y en un artículo del año 1966 adscribe explícitamente el modelamiento de la conducta hereditaria a las contingencias filogenéticas y el modelamiento de la conducta adquirida a las contingencias de la ontogenia. El primer proceso se explica por selección natural, el segundo, básicamente, por condicionamiento operante (cf. Skinner, 1988a, p. 385). Por cierto, la distinción entre selección natural, condicionamiento operante y selección de ambientes sociales podría ser irrelevante para la analogía fuerte, si la teo- 
ría del aprendizaje por condicionamiento operante y la teoría de la selección natural (los casos que aquí nos importan) fuesen meramente instancias de un mismo tipo de proceso natural. Pero, como mostraré, se trata de procesos cuya lógica explicativa es distinta, aunque Skinner las reúna a todas bajo el concepto de "selección por consecuencias" para distinguir estos procesos ciegos de selección, como advierte Richard Dawkins, de la selección consciente o intencional (cf. Skinner, 1988b; Dawkins, 1988, p. 33). Aunque Skinner asume que hay casos en donde los límites explicativos pueden ser difusos, claramente sostiene que se trata de teorías distintas que en determinados casos contribuyen conjuntamente a la explicación de determinadas conductas, como ocurre con el fenómeno de la impronta (imprinting). Respecto de esta conducta, Skinner (cf. 1976, p. 45) sostiene que lo heredado no es propiamente la respuesta de seguimiento por parte de los polluelos salidos del cascarón, sino más bien "la capacidad de ser reforzado manteniendo o reduciendo la distancia entre sí mismo y un objeto en movimiento" (Skinner, 1988a, p. 392). Pero este mismo fenómeno muestra, preliminarmente, que hay buenas razones para asumir, inicialmente, que Skinner piensa que la selección natural es una condición causal para la teoría del aprendizaje por condicionamiento operante y esta es, a su vez, una condición causal para la selección de ambientes sociales o para la evolución cultural (cf. Skinner, 1953, p. 430). Si la naturaleza explicativa de la teoría del condicionamiento operante es dependiente causalmente de la selección natural, una identificación fuerte entre estas teorías no puede ser sostenible, al menos nos encontramos aquí con procesos de distinto nivel y esto mostraría que la analogía fuerte debe ser abandonada. Pretender que ambas teorías son idénticas chocaría, además, con investigaciones empíricas que muestran el valor que tiene el aprendizaje por condicionamiento para preservar la capacidad de evolucionar por selección natural en determinadas especies. 5

Asumamos entonces que lo que Fodor y Piattelli-Palmarini quieren hacer es exclusivamente una analogía débil entre ambas teorías y que la analogía debería contribuir a mostrar la vacuidad explicativa de la explicación por selección natural. Los autores identifican seis constricciones que comparten la explicación conductista del aprendizaje y la explicación evolucionista: iteratividad, ambientalismo, gradualismo, monotonía, localidad y ausencia de causas mentales (FP, 2010, p. 5-11). No discutiré aquí ninguna de estas propiedades, me centraré en cambio en las afirmaciones que

5 Hay investigaciones empíricas que han mostrado el valor evolutivo del condicionamiento operante al mantener la variación genética disponible en una población conservando su tamaño o, alternativamente, constituyendo un mecanismo para suplir la falta de variación genética disponible en una población (cf. Brown, 2013, p. 944). Un ejemplo es la adquisición de la conducta de aversión del ratón marsupial (Planigale maculata) al sapo de caña (Rhinella marina), una especie invasora en Australia. Rachael Brown (cf. 2013, p. 936) atribuye al condicionamiento clásico o pavloviano la conducta adquirida del ratón marsupial de evitar esta posible presa cuya carne es venenosa, sin embargo, se trata claramente de un caso de condicionamiento de una conducta operante (la conducta de forrajeo). 
ellos hacen acerca del llamado "modelo de la caja negra" (FP, 2010, p. 4). Me parece que ninguna de esas constricciones explicativas, que comparten (parcialmente) la teoría conductista del aprendizaje y la teoría darwiniana, llegan a ser tan relevantes como la afirmación de que ambas teorías se comprometen con el modelo de la caja negra. De acuerdo con Fodor y Piattelli-Palmarini, considerar a una teoría como una teoría de caja negra es considerarla "simplemente como una función que mapea ciertas clases de inputs sobre ciertas clases de outputs" (FP, 2010, p. 3). Es decir, una función que relaciona las variables medioambientales con ciertos rasgos de los organismos.

Abrir la caja negra supone encontrar fenómenos de un nivel distinto, aunque causalmente relacionados con el nivel superior, que están determinados por otras leyes respecto de las que gobiernan los fenómenos que se estudian. Skinner se compromete con un modelo semejante cuando establece que la explicación de la conducta de un organismo cruza tres conjuntos interconectados de relaciones causales: un conjunto compuesto por la historia de causas que afectan desde el exterior a un organismo, el estado interno del organismo y la conducta del organismo (cf. Skinner, 1953, p. 34). Los estados internos no son relevantes para el análisis funcional conductista. Inicialmente, es suficiente con establecer que la conducta, el tercer conjunto de causas, es función del primer conjunto de causas.

Las variables externas, de las que la conducta es una función, proveen lo que podemos llamar una causa o un análisis funcional. Nos proponemos predecir y controlar la conducta del organismo individual. Esta es nuestra "variable dependiente", el efecto para el cual descubrimos una causa. Nuestras "variables independientes", las causas de la conducta, son las condiciones externas de las que la conducta es una función (Skinner, 1953, p. 35).

La irrelevancia de las causas fisiológicas para el análisis funcional no significa, sin embargo, que el organismo sea un receptáculo vacío o un recipiente cuyo contenido sea superfluo (cf. Skinner, 1976, p. 233). La investigación de la contribución que hace la biología del organismo a la generación de la respuesta conductual, permitiría, de acuerdo con Skinner, que "nos aproximáramos a una imagen más completa de la acción humana" (1976, p. 237). Debemos entender entonces que los estados internos no son relevantes para el análisis funcional conductista, pues la predicción y el control de la conducta no dependen argumentativamente de una explicación completa de los estados internos del organismo, es decir, estos estados internos no son parte del explanans de la explicación conductista (cf. Skinner, 19533, p. 60). Hay dependencia causal, pero la justificación para la predicción y el control conductual queda delimitada en la relación causal o funcional entre estímulos medioambientales y respuesta con- 
ductual. Mostraré que una de la razones de esta delimitación radica en que la naturaleza de esos estados internos del organismo no ha sido formada, según Skinner, originalmente, por los mecanismos conductistas de aprendizaje, sino por la selección natural.

Ahora bien, ¿cuál es, específicamente, la analogía que Fodor y Piattelli-Palmarini establecen con la explicación por selección natural? Ambas teorías tienen, de acuerdo con estos autores, exactamente la misma estructura explicativa, o sea, suponen variación azarosa o no dirigida y un mecanismo de filtro para esas variaciones. Las variaciones no dirigidas en la teoría darwiniana es variación genética y en la teoría conductista del aprendizaje es conducta operante generada aleatoriamente. Estos mismos puntos de semejanza entre ambas teorías, como he señalado, ya habían sido advertidos por el mismo Skinner (cf. 1953, p. 225; 1988a, p. 385). El resultado de la filtración de estas variaciones es evolución del rasgo en la teoría darwiniana y un nuevo perfil conductual en la teoría conductista del aprendizaje. De acuerd o con Fodor y Piattelli-Palmarini, la única diferencia relevante entre ambas teorías es que la variación genética es heredable y no así la variación conductual (FP, 2010, p. 16). Cuando abrimos la caja negra del conductismo, encontramos, de acuerdo con Fodor y Piattelli-Palmarini, las leyes de la asociación que proveen a Skinner una explicación de cómo las variables exógenas filtran los rasgos operantes para formar un nuevo patrón de conductas. Esas leyes de asociación resultan ser, sin embargo, falsas. No ocurre lo mismo con la teoría darwiniana. Fodor y Piattelli-Palmarini sostienen que la teoría de la evolución "no puede ofrecer ninguna explicación remotamente plausible de cómo el filtro de la selección natural podría trabajar" (FP, 2010, p. 16). Cuando abrimos la caja negra de Darwin, no encontramos nada: la teoría de Darwin es una teoría vacía.

\section{TRASFONDO GAUSAL Y GONDIGIONAMIENTO DE OPERANTES}

Hay razones conceptuales por las cuales la analogía fracasa. Ciertamente, somos libres para establecer la analogía que queramos, pero no parece razonable establecer una analogía para intentar refutar una teoría cuando las teorías a comparar son acerca de fenómenos que no son causalmente independientes, dado el tipo de dependencia lógica que puede generarse, como he sostenido, entre estas teorías. He mostrado que hay evidencias preliminares de naturaleza historiográfica que indican que el trasfondo causal que encontramos en la caja negra de la teoría del aprendizaje por condicionamiento operante es un trasfondo biológico modelado por la teoría darwiniana. No se trata aquí meramente de un antecedente histórico o de un contexto cultural en el que se inserta la teoría del condicionamiento operante. La teoría de la selección natural constituye una condición que hace históricamente posible el surgimiento de la teoría del condi- 
cionamiento operante. Como he dicho Fodor y Piattelli-Palmarini ignoran la relación de dependencia causal y eluden así el tipo de relación lógica que se establece entre ambas teorías. En su reemplazo atribuyen a Skinner un compromiso con un trasfondo explicativo de reglas de asociación heredadas del empirismo británico, haciendo al condicionamiento operante, de esta manera, causalmente independiente de la teoría darwiniana. Pero esta maniobra es conceptual e históricamente inaceptable. La teoría conductista no está comprometida con los principios mentales de asociación pre-darwinianos de Locke o de Hume mediante los cuales las ideas tenderían a formar necesariamente ciertas asociaciones (cf. Locke, 1959, 2, vi; Hume, 1978, p. 399). Los antecedentes de la teoría conductista del aprendizaje no se encuentran en el asociacionismo mentalista del empirismo británico, sino en el trabajo experimental y teórico que Thorndike presentara en su informe de investigación, Animal intelligence, de 1898. Charles Taylor ha destacado la relevancia histórica que tiene este estudio para el surgimiento de la teoría conductista del aprendizaje (cf. Taylor, 1964). Taylor se refiere particularmente a la Ley del Efecto de Thorndike, de acuerdo con la cual el vínculo entre las respuestas de un animal a determinada situación se ve fortalecido o debilitado según sean sus consecuencias satisfactorias o molestas, respectivamente (cf. Thorndike, 1911, p. 244). De acuerdo con Taylor, la Ley del Efecto "cortó el nudo gordiano" con el que el empirismo británico había unido la conducta humana y las causas mentalistas. Dice Taylor:

Para el viejo punto de vista la acción estaba gobernada por el placer y el dolor, esto es, lo que los humanos y otros organismos animados buscan y evitan. El valor para la sobrevivencia de este proceso radica, por un lado, en la afortunada congruencia de las actividades placenteras y las actividades necesarias para el organismo y, por otro, entre las experiencias dolorosas y las experiencias nocivas para el organismo. (...) La explicación de esta congruencia es variada. Locke, por ejemplo, la atribuye a la sabiduría y la generosidad de Dios. Cuando el problema de la adaptación realmente tomó relevancia con los descubrimientos de Darwin, la tendencia fue explicar la congruencia por selección natural (Taylor, 1964, p. 116).

Taylor agrega que aunque la Ley del Efecto incluye los términos mentalistas de "satisfacción" y "malestar", el contenido específico de estos términos es abandonado en favor de la conexión empírica entre estímulos y respuestas. Skinner coincide explícitamente: "Comúnmente se dice que algo es reforzado porque se siente, luce, suena, huele o sabe bien, pero desde el punto de vista de la teoría evolucionista una susceptibilidad al reforzamiento es debida a su valor para la sobrevivencia y no a algún sentimiento asociado" (Skinner, 1976, p. 52). 
Lo que ha identificado Taylor es una condición que ha hecho históricamente posible el surgimiento de la teoría conductista, sin la explicación causal que proporciona la teoría de la selección natural, para dar cuenta en términos naturalistas y no mentalistas de esas conexiones que identifica la Ley del Efecto, la teoría del condicionamiento operante no hubiera sido posible tal como la conocemos. Aunque no es del todo correcto establecer una conexión exclusiva entre la Ley de Efecto y el principio de selección natural, hay buenas razones para sostener que la conexión radica de manera primaria en lo que Thorndike llama "la ley de la conducta ancestral" (the law of original behavior) o también "ley del instinto". De acuerdo con esta ley, "en cualquier situación un animal, además de aprender, responderá en virtud de la naturaleza inherente de sus sistemas de recepción, conexión y acción” (Thorndike, 1911, p. 243). Thorndike nos advierte que sus experiencias con animales las interpreta "en términos de las conexiones ancestrales (originals) y adquiridas entre situación y respuesta" (Thorndike, p. v). La menos citada ley del instinto está entonces en un nivel diferente de las dos leyes de la adquisición de la conducta o leyes del aprendizaje, es decir, la ley del efecto y la ley del ejercicio, ${ }^{6}$ pero no por ello es menos relevante para la explicación conductista. La ley del instinto describe mecanismos conductuales modelados por procesos ancestrales de selección natural:

La evolución de la sensibilidad y de los sistemas de acción de los animales ha sido ya objeto de estudio de naturalistas. La evolución del sistema de conexión lo será pronto. Cada reflejo, instinto o capacidad, cada vínculo entre, por un lado, una situación determinada que se presenta a un estado fisiológico determinado y, por otro, una respuesta determinada, tiene su árbol genealógico (ancestral tree) (Thorndike, 1911, p. 275).

Thorndike hace una clara distinción teórica entre el mecanismo de selección natural que está involucrado en la formación de las respuestas que describe la ley del instinto y los mecanismos de aprendizaje que describen la ley del efecto y la ley del ejercicio. Aunque también advierte acerca de la relación de dependencia causal entre estos procesos. Los hechos, nos dice, "del intelecto y del carácter que son explicados por el aprendizaje, que no son propiedades inherentes de las especies y consecuentemente están más allá del alcance de la evolución en el linaje (in the race), no estuvieron separados de los hechos de la naturaleza original" (Thorndike, 1911, p. ${ }_{2} 3$ ). No sería posible la adquisición de nuevas conductas por los mecanismos que describe la ley del

6 La ley del ejercicio nos dice: “cualquier respuesta a una situación será, si todo lo otro sigue igual, más fuertemente conectada con la situación en proporción al número de veces que ha sido conectada con esa situación y el promedio del vigor y duración de las conexiones" (Thorndike, 1911, p. 244). 
efecto, si de manera previa el organismo no estuviera equipado con respuestas originales o ancestrales. Esto muestra la dependencia de la explicación conductista de una teoría de trasfondo que proporcione una explicación causal de esas respuestas originales. Pero no se trata aquí de una explicación funcional o fisiológica, sino de una explicación en términos de lo que Ernst Mayr identificó como causas últimas, es decir, en términos de lo que explica que esta respuesta haya surgido en poblaciones ancestrales (cf. Mayr, 1961, p. 1503). La teoría darwiniana sostiene que esta explicación reside, básicamente, en procesos de selección natural.

La dependencia que tiene la ley del instinto de Thorndike de la explicación darwiniana puede verse con claridad en una noción que le es simétrica: el espaciamiento innato de cualidades de Quine.

Un patrón de similaridad es, en algún sentido, innato. Este punto no va en contra del empirismo; es un lugar común de la psicología conductista. Una respuesta a un círculo rojo será, si es recompensada, producida nuevamente por una elipse rosa más prontamente que por un triángulo azul; el círculo rojo se parece más a la elipse rosa que al triángulo azul. Sin tal espaciamiento previo de cualidades no podríamos adquirir un hábito; todos los estímulos serían igualmente parecidos e igualmente diferentes (Quine, 1969, p. 123).

El mismo concepto de refuerzo carecería de sentido para la teoría conductista del aprendizaje sin este espaciamiento innato de cualidades. Para que una conducta pueda ser reforzada el sujeto "tiene que sentir más parecido entre ciertas estimulaciones que entre otras" (Quine, 1969, p. 82). Quine asume explícitamente que el espaciamiento innato de cualidades se explica por mecanismos de selección natural (cf. Quine, 1969, p. 126).

Skinner, como he señalado, advirtió la semejanza entre la teoría del condicionamiento operante y la selección natural, pero, a diferencia de Fodor y Piattelli-Palmarini, vio claramente en donde radican las diferencias entre ambas teorías. La selección natural explica los rasgos de una especie o de una población, la teoría del condicionamiento operante explica los rasgos conductuales de los organismos individuales. Pero hay algo más, se trata aquí de teorías que están en niveles distintos aunque estrechamente relacionados. La teoría del condicionamiento operante se apoya en la teoría de la selección natural y Skinner lo dice explícitamente:

El análisis conductual descansa en el supuesto siguiente: una persona es primero que nada un organismo, un miembro de una especie y una subespecie, que posee una dotación genética de rasgos anatómicos y fisiológicos, los que son productos 
de las contingencias de la sobrevivencia a las que las especies han sido expuestas en el proceso de la evolución. El organismo llega a ser una persona cuando adquiere un repertorio de conductas bajo las contingencias del reforzamiento al que es expuesto durante su vida (Skinner, 1976, p. 19).

Skinner estuvo en lo correcto aquí, el desarrollo de los rasgos del organismo individual, su ontogenia, no determina la evolución de la población. La explicación por selección es una explicación de las propiedades de una especie, de la frecuencia de los rasgos en una población, y no explica los rasgos de los organismos individuales. Este es el carácter no distributivo de la explicación por selección (cf. Sober, 1984; Razeto \& Frick, 2011). En la teoría evolucionista, la explicación de los rasgos de un individuo es una explicación por desarrollo, no una explicación por selección (cf. Sober, 1984; Sober, 1995). La explicación por desarrollo es la explicación de una propiedad de un individuo en términos de los mecanismos o condiciones internas al individuo que dan cuenta de la naturaleza de sus rasgos en la interacción con determinado ambiente. La teoría del aprendizaje por condicionamiento operante considerada como un tipo de explicación adaptativa, constituiría, por cierto, un tipo de adaptación no darwiniana y correspondería a lo que Lewontin identificó como una teoría de evolución transformacional (cf. Lewontin, 1983). En los procesos de aprendizaje por condicionamiento operante una población cambia su perfil conductual porque los individuos que la componen han cambiado su perfil conductual.

\section{Conclusiones}

He mostrado el fracaso del intento de Fodor y Piattelli-Palmarini por desacreditar la teoría evolucionista por medio de una analogía entre selección natural y condicionamiento operante. De acuerdo con ellos, la persistencia de la teoría de la selección natural se explica justamente porque los evolucionistas no se han percatado de esta analogía y no han percibido que los mismos argumentos que refutaron la teoría del condicionamiento operante refutan la teoría de la selección natural. Fodor y Piattelli-Palmarini cuentan con que el evidente desprestigio que actualmente tiene la teoría conductista del aprendizaje contamine a la teoría de la selección natural y, al parecer, se ven a sí mismos, en la refutación de la teoría darwiniana, cumpliendo un papel semejante al que tuvo Chomsky en el desmantelamiento del programa conductista de Skinner. Esa estrategia podría quizás tener ciertas posibilidades de éxito inicial si realmente la lógica explicativa de ambas teorías fuera semejante. Pero, aunque ese fuera el caso, el argumento está condenado desde un inicio a la ruina porque, según traté de mostrar, el 
proceso de condicionamiento operante supone como condición causal al proceso de selección natural. Fodor y Piattelli-Palmarini niegan esto y pretenden que el trasfondo causal de la teoría del condicionamiento operante estaría constituido por los principios de asociación de ideas que los conductistas heredarían del empirismo británico. Pero esa tesis no solo es falsa, sino que es anacrónica, ya que desarticula a la teoría de las condiciones históricas que la hicieron posible. He identificado dos de esas condiciones: (1) La teoría de la selección natural proporciona por primera vez, como lo advirtió Taylor, una explicación causal naturalista y no mentalista de la congruencia entre, por un lado, el placer y las conductas necesarias para los organismos y, por otro, el dolor y las conductas nocivas. Sin esa explicación causal el programa conductista de Skinner no hubiera sido posible o, al menos, hubiese sido muy distinto; y (2) la teoría de la selección natural proporciona una explicación causal última de la ley del instinto de Thorndike y de lo que Quine llamó "espaciamiento previo o innato de cualidades". Este espaciamiento es una condición necesaria para la generación del hábito o del condicionamiento. La explicación causal de nuestra tendencia a ser condicionado no la proporciona la teoría del condicionamiento, esta explicación proviene de la teoría de la selección natural. ¿Cómo Fodor y Piattelli-Palmarini pudieron ignorar estas condiciones históricas que proporciona la teoría de la selección natural dada la proliferación de referencias a la teoría darwiniana que encontramos en los escritos de los conductitas? Bien, mi explicación es que esto no tiene que ver con una determinada comprensión de la lógica de la explicación conductista, sino básicamente se trata de una motivación externa que proviene de su decisión de emular la refutación de Chomsky al conductismo como teoría del aprendizaje: si hubiesen aceptado la vinculación causal tendrían que haber aceptado que la teoría de la selección natural es una condición necesaria de la teoría del condicionamiento operante y que, por tanto, pretender refutar la teoría de la selección natural a través de la refutación de la teoría del condicionamiento operante generaría una falacia de la afirmación del consecuente. Pero una vez eludida, sobre la base de premisas erróneas, esa relación de condición necesaria, todo el peso del argumento queda apoyado en la tesis según la cual la lógica explicativa entre ambas teorías es básicamente la misma. La apuesta inicial es por una identificación fuerte entre ambas teorías, pero prontamente abandonan esta idea, las diferencias resultan explícitas y ellos mismos presentan las primeras evidencias. Pero la analogía débil tampoco funciona. A pesar del énfasis que Fodor y Piattelli-Palmarini ponen acerca de que la explicación por selección tiene ver con la explicación de los rasgos de un organismo, la teoría evolutiva no hace esto, la teoría de la selección natural es una explicación de la frecuencia de rasgos al interior de una población, específicamente, ofrece una explicación de cómo evolucionan en la historia de una población los rasgos en 
covariación con determinadas condiciones medioambientales. Skinner, a diferencia de Fodor y Piattelli-Palmarini, pudo ver con claridad esta diferencia cuando adscribió el origen de la conducta hereditaria a las contingencias filogenéticas y el origen de la conducta adquirida a las contingencias de la ontogenia.@

\title{
Julio Torres Meléndez
}

Departamento de Filosofía, Universidad de Concepción, Chile. jutorres@udec.cl

\section{Natural selection and operative conditioning: a critique of Fodor and Piatelli-Palmarini's analogy}

\begin{abstract}
ABSTRAGT
In this paper I argue against the analogy between natural selection and operant conditioning that Fodor and Piattelli-Palmarini used to argue that the same arguments that discredit the behaviorist theory discredit the theory of natural selection. These theories are not independent either conceptually or historically and, therefore, it is unreasonable to make an analogy between selection and operant conditioning that can serve Fodor and Piattelli-Palmarini's intentions. Natural selection is a causal condition of the mechanism of operant conditioning, so the refutation of the theory of operant conditioning cannot be a reason for the refutation of the theory of natural selection.
\end{abstract}

KeYwords $・$ Natural selection. Operant conditioning. Skinner. Fodor. Piattelli-Palmarini.

\section{REFERENGIAS BIBLIOGRÁFICAS}

Bıock, N. (Org.). Readings in philosophy of psychology. Cambridge: Harvard University Press, 1980 [1959]. V. 1.

BRown, R. L. Learning, evolvability and exploratory behaviour: extending the evolutionary reach of learning. Biology and Philosophy, 28, p. 933-55, 2013.

Catania, A. C. \& Harnad, S. (Ed.). The selection behavior. The operant behaviorism of B. F. Skinner: comments and consequences. Cambridge: Cambridge University Press, 1988.

Сномsкy, N. A review of B. F. Skinner's verbal behavior. In: Bцock, N. (Org.). Readings in philosophy of psychology. Cambridge: Harvard University Press, 1980 [1959].v. 1, p. 48-63.

DAWKINS, R. Replicators, consequences, and displacement activities. In: CatANIA, A. C. \& HARnAD, S. (Ed.). The selection behavior. The operant behaviorism of B.F. Skinner: comments and consequences. Cambridge: Cambridge University Press, 1988. p. 34-5.

Diéguez, A. La vida bajo escrutinio. Una introducción a la filosofía de la biología. Barcelona: Biblioteca Buridán, 2012. 
Díez, J. \& Lorenzano, P. Who got what wrong? Fodor and Piattelli on Darwin: guiding principles and explanatory models in natural selection. Erkenntnis, 78, p. 114,3-75, 2013.

Fodor, J. \& Piattelli-Palmarini, M. What Darwin got wrong. New York: Farrar/Straus/Giroux, 2010. (FP) Godfrey-Sмith, P. It got eaten. Review of "What Darwin got wrong by Fodor, J. \& Piattelli-Palmarini, M.”. London Review of Books, 32, 13, p. 29-30, 2010.

Gould, S. J. La falsa medida del hombre. Barcelona: Crítica, 2009 [1981].

Hume, D. A treatise of human nature. Oxford: Clarendon Press, 1978 [1739].

Lewontin, R. Darwin's revolution. The New York Review of Books, 3o, p. 21-7, 1983.

Locke, J. An essay of concerning human understanding. New York: Dover, 1959 [169o].

MaYr, E. Cause and effect in biology. Science, 134, 34.89, p. 1501-6, 1961.

Piattelli-Palmarini, M. et al. Ofminds and language: a dialogue with Noam Chomsky in the basque country. Oxford: Oxford University Press, 2009.

Quine, W. V. Ontological relativity and other essays. New York: Columbia University Press, 1969.

RAzeto, P. B. \& Frick, R. Probabilistic causation and the explanatory role of natural selection. Studies in History and Philosophy of Biological and Biomedical Sciences, 42, 3, p. 344,-55, 2011.

Soвer, E. The nature of selection. Evolutionary theory in philosophical focus. Chicago: The University of Chicago Press, 1984 .

. Natural selection and distributive explanation: a reply to Neander. British Journal for the Philosophy of Science, 46, p. 384-97, 1995 .

. Natural selection, causality, and laws: what Fodor and Piatelli-Palmarini got wrong. Philosophy of Science, 77, p. 594-607, 2010.

Skinner, B. F. Science and human behavior. New York: The Free Press, $195^{3}$. About behaviorism. New York: Vintage Books, 1976 [1974].

The phylogeny and ontogeny of behavior. In: Catania, A. C. \& Harnad, S. (Ed.). The selection behavior. The operant behaviorism of B.F. Skinner: comments and consequences. Cambridge: Cambridge University Press, 1988a. p. 382-4,00.

. Selection by consequences. In: Catania, A. C. \& Harnad, S. (Ed.). The selection behavior. The operant behaviorism of B.F. Skinner: comments and consequences. Cambridge: Cambridge University Press, 1988b [1981].p. 11-20.

TAYLOR, Ch. The explanation of behaviour. London: Routledge and Kegan Paul, 1964.

Thorndike, E. L.Animal intelligence. Experimental studies. New York: The Macmillan Company, 1911.

Wittgenstein, L. Investigaciones filosóficas. México: UNAM, 2003.

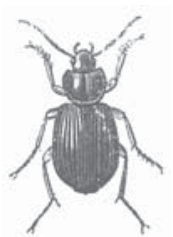

